\title{
(2) Long COVID following mild SARS-CoV-2 infection: OPEN ACCESS characteristic $T$ cell alterations and response to antihistamines
}

\author{
Paul Glynne, ${ }^{1}$ Natasha Tahmasebi, ${ }^{2}$ Vanya Gant, ${ }^{3}$ Rajeev Gupta (1) 4,5
}

Additional supplemental material is published online only. To view, please visit the journal online (http://dx. doi.org/10.1136/jim-2021002051)

${ }^{1}$ The Physicians Clinic, London, UK

${ }^{2}$ King's College London School of Medicine, London, UK

${ }^{3}$ Departments of Infection and Microbiology, University College London Hospitals NHS Foundation Trust,

London, UK

${ }^{4}$ Stem Cell Laboratory, UCL Cancer Institute, London, UK ${ }^{5}$ Manual Blood Sciences, Health Services Laboratories, London, UK

\section{Correspondence to} Dr Rajeev Gupta, Stem Cell Laboratory, UCL Cancer Institute, London, UK: rajeev.gupta@ucl.ac.uk

Accepted 25 August 2021 Published Online First 5 October 2021

\begin{abstract}
Long COVID is characterized by the emergence of multiple debilitating symptoms following SARS-

CoV-2 infection. Its etiology is unclear and it often follows a mild acute illness. Anecdotal reports of gradual clinical responses to histamine receptor antagonists (HRAs) suggest a histamine-dependent mechanism that is distinct from anaphylaxis, possibly mediated by $T$ cells, which are also regulated by histamine. T cell perturbations have been previously reported in post-viral syndromes, but the T cell landscape in patients who have recovered from mild COVID-19 and its relationship to both long COVID symptoms and any symptomatic response to HRA remain underexplored. We addressed these questions in an observational study of 65 individuals who had recovered from mild COVID-19. Participants were surveyed between 87 and 408 days after the onset of acute symptoms; none had required hospitalization, 16 had recovered uneventfully, and 49 had developed long COVID. Symptoms were quantified using a structured questionnaire and $T$ cell subsets enumerated in a standard diagnostic assay. Patients with long-COVID had reduced CD4+ and CD8+ effector memory (EM) cell numbers and increased PD-1 (programmed cell death protein 1) expression on central memory (CM) cells, whereas the asymptomatic participants had reduced CD8+ EM cells only and increased CD28 expression on CM cells. $72 \%$ of patients with long COVID who received HRA reported clinical improvement, although T cell profiling did not clearly distinguish those who responded to HRA. This study demonstrates that $T$ cell perturbations persist for several months after mild COVID-19 and are associated with long COVID symptoms.
\end{abstract}

\section{INTRODUCTION}

As of August 2021, there have been in excess of 200 million cases of COVID-19 worldwide, with more than 4.4 million deaths (https://coronavirus.jhu.edu/map.html). Not all patients surviving the acute infection recover rapidly and uneventfully. Initial resolution of the acute illness may be followed by a combination of clinical sequelae, including but not limited to pulmonary, neurologic, dermatologic, cardiac, renal, endocrine and autoinflammatory phenomena, collectively described as long

\section{Significance of this study}

What is already known about this subject?

- Twenty per cent of patients who suffer clinically mild SARS-CoV-2 infection may develop long COVID.

- Symptoms may persist for several months.

- The etiology is unclear.

What are the new findings?

- This observational study demonstrates clear symptomatic improvement in response to combined $\mathrm{H} 1 / \mathrm{H} 2$ receptor blockade.

- Long COVID is associated with characteristic and specific alterations in circulating $T$ cells that persist for up to 400 days after the initial COVID-19 infection.

How might these results change the focus of research or clinical practice?

- These data encourage future randomized trials of antihistamines in long COVID.

- T cell immunophenotyping may provide a rapid and high-throughput diagnostic test for long COVID.

- Prospective isolation and molecular analysis of $\mathrm{T}$ cells from patients with long COVID may provide further insights into the pathogenesis of this and perhaps other post-viral syndromes.

COVID. ${ }^{1}$ Importantly, such symptoms may develop following apparent recovery from an initially mild acute illness that did not require medical intervention. It is not uncommon for at least one symptom to persist up to and beyond 7 months. $^{2}$

The majority of patients presenting with long COVID will not have been hospitalized or sustained significant end-organ damage during their initial illness. In contrast to acute COVID19 , it predominantly affects younger patients with few comorbidities, who had relatively mild initial infectious symptoms and who did not come to medical attention until their long COVID symptoms appeared. It is expected to place increasing burdens on healthcare systems, and by delaying the return of these often working people to normal life, it will have wider societal and economic impacts. ${ }^{34}$ 
Long COVID shares many features with other postviral and idiopathic chronic fatigue syndromes. ${ }^{1}$ Persistent alterations both in the numbers of various $\mathrm{T}$ cell subtypes and their biological properties have been reported in such disorders. ${ }^{5}{ }^{6}$ In acute COVID-19, perturbations in both B and $\mathrm{T}$ cells are observed irrespective of disease severity. ${ }^{78}$ Studies of T cells from individuals who have recovered from COVID-19 have thus far focused on antigen specificity, primarily to understand cellular immunity to SARSCoV-2. ${ }^{79}$ Beyond this, a detailed survey of any immunologic sequelae that may persist following clearance of SARS-CoV-2 and how they might relate to a cohort of long COVID sufferers is lacking.

While some patients presenting with long COVID, particularly those who were hospitalized during their initial illness, will have clinical signs and abnormal blood or other tests, the majority do not, and there are currently no validated laboratory tests for the condition. Objective diagnostic criteria and treatment strategies are urgently required, especially as attempts to better define it remain suboptimal. ${ }^{41}$ Currently, several key clinicopathological questions are unanswered, specifically (1) why those individuals who go on to develop long COVID do so; (2) the pathological mechanisms responsible for it; and (3) the rationale for, and efficacy of, candidate therapies.

To address these clinical imperatives, we report here the findings of a preliminary observational study undertaken in a single medical outpatient clinic to (1) describe the range of clinical symptoms in a cohort of patients presenting with long COVID; (2) investigate the possible benefit of HRA on long COVID-19 symptoms; and (3) interpret these in the light of simultaneous peripheral blood flow cytometry analysis, focusing on the numbers and phenotype of cells important to acquired antiviral immunity. All patients in the study had a mild initial infection — none had required hospitalization for acute COVID-19, and none had received prior immunomodulatory therapy. Volunteers who had uneventfully and rapidly recovered from proven COVID-19 infection were recruited as controls.

\section{METHODS}

\section{Study setting}

The Physicians' Clinic (TPC; part of HCA healthcare UK, the study sponsor) is a private outpatient and diagnostic center in London.

\section{Study design}

This is a prospective observational study of patients previously diagnosed with mild COVID-19 who having initially recovered, subsequently developed persistent protean symptoms suggestive of long COVID. Participants were recruited between November 2020 and April 2021. None had previously sought medical attention or 'treatment' for acute COVID-19, none had a history of autoimmunity, and none had received immunomodulatory medications.

We recruited 49 patients with long COVID ('symptomatic group', symptoms >84days following acute COVID-19 infection; physician or laboratory diagnosis) to undergo blood sampling to measure several hematologic and biochemical variables, and for flow cytometry. The tests were also offered to 16 volunteer clinician colleagues, all of whom had either PCR or serological evidence of COVID-19 and who had recovered uneventfully ('asymptomatic group'). The age and gender distributions of the two groups were similar.

A symptom questionnaire was designed with a binary symptom grid to initially record the presence (score 1 ) or absence (score 0 ) of the following long COVID symptom categories: fatigue, constitutional upset (sweats, fever, arthralgia, myalgia), breathlessness, post-exertional malaise (PEM), chest pain, neurologic (headaches, neurosensory, brain fog), neuropsychiatric (anxiety, insomnia), dysautonomia (postural tachycardia), ear, nose and throat symptoms, gastrointestinal disturbance (food intolerance, diarrhea, bloating), and dermatological manifestations (rashes, flushing, urticaria), to give a maximum possible symptom score of 11 . The questionnaires were collated by one of us (NT) who was not involved in the management of the patients, and blinded to their clinical details.

Initially, patients were offered supportive care only. However, as the study progressed, all patients were offered empiric treatment trials with a combination of H1 (loratadine $10 \mathrm{mg}$ two times per day or fexofenadine $180 \mathrm{mg}$ two times per day) and $\mathrm{H} 2$ (famotidine $40 \mathrm{mg}$ once daily or nizatidine $300 \mathrm{mg}$ once daily) receptor antagonists (HRAs) for a minimum of 4 weeks as part of their ongoing care. Of the 49 long COVID-19 study participants, 26 patients consented to try HRA. Between 4 and 16 weeks after starting treatment, both HRA-treated and untreated patients were asked to grade their symptoms as now absent, identical, better, or worse (online supplemental figure 1). In this analysis, 'absent' or 'better' scored 0 , and 'identical' or worse, 1.

\section{Flow cytometry and additional laboratory measurements} This was performed on peripheral blood collected in EDTA. The Beckman Coulter TQ-prep whole blood lysis system (Beckman Coulter Life Sciences, High Wycombe, UK) was used to prepare cells for flow cytometry. Antibody staining was with the Duraclone IM T lyophilized antibody panel (B53328, Beckman Coulter) as described previously. ${ }^{12}$ This is in routine diagnostic use in our clinical practice, and normal ranges had been previously established (in 2018) in healthy adults with normal automated blood counts for accreditation purposes. Analysis of primary flow cytometry data was in Kaluza C (Beckman Coulter) and was undertaken by one of us $(\mathrm{RG})$, who was blinded to clinical information collected and collated by others. The gating strategy and phenotypes analyzed are shown in online supplemental figure 2. An automated full blood count was performed on every specimen in parallel to flow cytometry. T cell populations were quantified as a percentage of total cellularity, and absolute numbers calculated from the corresponding total white cell count. Single cell antigen density was recorded as median fluorescence intensity (MFI).

\section{Statistical analysis}

Comparison of clinical data from the asymptomatic and symptomatic groups was by Mann-Whitney t-test. Comparison of response to HRA was by Wilcoxon matched pairs signed-rank test. All flow cytometry fluorescence data and numbers of $T$ cell subpopulations were assumed to lie in non-Gaussian distributions, and analysis of variance was by 
Table 1 Clinical features of study participants

\begin{tabular}{|c|c|c|}
\hline Clinical characteristics & $\begin{array}{l}\text { Long COVID } \\
\text { (symptomatic, } n=49 \text { ) }\end{array}$ & $\begin{array}{l}\text { Post-COVID controls } \\
\text { (asymptomatic, } n=16 \text { ) }\end{array}$ \\
\hline Age range (median) & $25-65(43)$ & $25-72(34.5)$ \\
\hline Female (\%) & $30(61.2 \%)$ & $8(50 \%)$ \\
\hline \multicolumn{3}{|l|}{ Ethnicity } \\
\hline White & $45(91.8 \%)$ & $12(75 \%)$ \\
\hline Asian & $2(4.1 \%)$ & $2(12.5 \%)$ \\
\hline Black & $1(2 \%)$ & $1(6.3 \%)$ \\
\hline Mixed & $1(2 \%)$ & $1(6.3 \%)$ \\
\hline $\begin{array}{l}\text { Comorbidities } \\
1 \text { cancer, } 1 \text { controlled } \\
\text { hypertension }\end{array}$ & $2(4.1 \%)$ & 0 \\
\hline Allergy or atopy & $16(32.7 \%)$ & $1(5.8 \%)$ \\
\hline $\begin{array}{l}\text { Mean days from acute COVID } \\
\text { to study testing }\end{array}$ & 271.8 days & 321.6 days \\
\hline $\begin{array}{l}\text { Vaccination history at time of } \\
\text { recruitment (at least one } \\
\text { dose) }\end{array}$ & $1 / 49(2.0 \%)$ & $14(87.5 \%)$ \\
\hline $\begin{array}{l}\text { SARS-CoV-2 antibodies } \\
\text { detected }\end{array}$ & $20 / 49(40.8 \%)$ & $13 / 16(81.3 \%)$ \\
\hline \multicolumn{3}{|l|}{ Long COVID symptoms } \\
\hline Fatigue & $36(73.5 \%)$ & Not applicable \\
\hline Constitutional & $31(63.3 \%)$ & Not applicable \\
\hline Breathlessness & $19(38.8 \%)$ & Not applicable \\
\hline Post-exertional malaise & $35(71.4 \%)$ & Not applicable \\
\hline Chest pain (non-cardiac) & $18(36.7 \%)$ & Not applicable \\
\hline Neurologic/neurosensory & $39(79.6 \%)$ & Not applicable \\
\hline Neuropsychiatric & $29(59.2 \%)$ & Not applicable \\
\hline $\begin{array}{l}\text { Dysautonomia (postural } \\
\text { tachycardia syndrome) }\end{array}$ & $14(28.6 \%)$ & Not applicable \\
\hline Ear, nose and throat & $22(44.9 \%)$ & Not applicable \\
\hline Gastrointestinal & $20(40.8 \%)$ & Not applicable \\
\hline Dermatological & $23(46.9 \%)$ & Not applicable \\
\hline
\end{tabular}

Kruskal-Wallis H test. Dunn's multiple comparison test was then used to estimate statistical significance. All statistical analysis, including principal component analysis, was in Prism V.9.1 (GraphPad Software, LLC).

\section{RESULTS}

\section{Patient characteristics}

Forty-nine patients with a diagnosis of long COVID were recruited. Twenty-five had either PCR or serological evidence for COVID-19. The remainder had suffered their acute illnesses at the start of the pandemic, when PCR testing was not widely available in the UK, and had not subsequently returned positive serology tests. Only one had been vaccinated at the time of enrollment into the study. We recruited 16 individuals known to have had acute COVID-19 infection but who had recovered rapidly and uneventfully to serve as asymptomatic controls. The majority in this group are healthcare professionals; all had had either positive PCR tests or serology, and 14 had received at least one vaccination dose (Pfizer) at the time of enrollment. Participants' baseline clinical features are shown in table 1 .

Almost all patients with long COVID were polysymptomatic $(95.8 \%)$ as described by others ${ }^{13}$ with an average of 4.58/11 typical symptoms (range 1-10). The average symptom duration was 268.9 days (range $87-402$ ) at the time of referral and participation in the study. Patients with long COVID were relatively young (mean 43; range 25-65 years) and showed a female preponderance (29/49; $60.4 \%)$. Seventeen out of 49 patients with long COVID had a history of atopy, which is in keeping with reports that atopy is predictive of mild acute disease. ${ }^{14}$

\section{Routine blood tests}

These were undertaken at first presentation to our clinic and included full blood count, erythrocyte sedimentation rate (ESR), $\mathrm{C}$ reactive protein (CRP), D-dimer, renal and liver function tests. The results were within the normal range in almost every case. Two out of 49 patients had mildly elevated CRP (NR $=<5 \mathrm{mg} / \mathrm{L})$ and 4 out of 49 had a mildly elevated ESR ( $\mathrm{NR}=<15 \mathrm{~mm} /$ hour). Circulating total and differential leukocyte numbers were normal in all patients; one patient had an incidental borderline normocytic anemia.

\section{Treatment with HRA}

At first presentation, symptoms were categorized using the symptom grid. Twenty-six patients (16 female, 10 male, mean age 44 years) were treated empirically with HRA, and 23 (14 female, 9 male, mean age 41) either declined HRA or were not offered them because they were first seen before HRA treatment became part of our practice. All patients were offered standard supportive care and advice to control symptoms (NICE guideline NG188: https://www.nice.org. uk/guidance/ng188/RCGP/SIGN guidelines).

The symptom profiles in the treated and untreated cohorts were similar (table 2 ), the mean symptom burdens were 4.28/11 (range 1-8/11) and 4.91/11 (range 1-10/11). In the treated group, HRA reduced average symptom to 2.68/11 (range 0-6/11) (table 2, figure 1A). The mean time to response was 29.6 days (median 26 days; range 6-89 days). Nineteen patients $(72 \%)$ experienced a reduction in their symptoms: $5(20 \%)$ reported complete resolution of all symptoms, 13 (52\%) reported some improvement, 6 reported no change, and 1 deteriorated (developing PEM and insomnia shortly after starting loratadine and famotidine). Patients reported improvements in all symptoms except dysautonomia (table 2). Only 9 of the 19 patients who responded to HRA had detectable SARS-CoV-2 antibodies. Of the 17 patients with long COVID with a history of atopy, 11 received HRA, and of these, 8 reported a clinical improvement.

The 23 patients who did not receive HRA were also reassessed between 28 and 119 days after their initial blood tests (median 56 days). Twenty-six per cent reported some spontaneous improvement in their symptoms. One experienced a full recovery, and in five, there was a reduction in some but not all symptoms. However, the majority $(15,65 \%)$ reported no change, and $2(9 \%)$ developed new additional symptoms (figure 1B). There was no correlation between SARS-CoV-2 antibody status and spontaneous resolution of symptoms in this group.

\section{T cell compartments}

Flow cytometry was performed once as part of the initial blood tests. Despite the time that had elapsed from the acute COVID-19 illness (see table 1, long COVID symptomatic 
Table 2 Symptom profiles of treated and untreated cohorts

\begin{tabular}{|c|c|c|c|c|}
\hline \multirow[b]{3}{*}{ Symptom group } & \multicolumn{4}{|c|}{ Number of patients reporting symptom } \\
\hline & \multicolumn{2}{|l|}{ HRA treated (26) } & \multicolumn{2}{|l|}{ Untreated (23) } \\
\hline & Initial assessment & Follow-up assessment & Initial assessment & Follow-up assessment \\
\hline Fatigue & $17 / 26$ & $12 / 26$ & $15 / 23$ & $15 / 23$ \\
\hline Constitutional upset & $10 / 26$ & $7 / 26$ & $16 / 23$ & $14 / 23$ \\
\hline Breathlessness & $6 / 26$ & $5 / 26$ & $9 / 23$ & $8 / 23$ \\
\hline Post-exertional malaise & $12 / 26$ & $11 / 26$ & $16 / 23$ & $14 / 23$ \\
\hline Chest pain & $13 / 26$ & $6 / 26$ & $6 / 23$ & $5 / 23$ \\
\hline Neurologic & $13 / 26$ & $4 / 26$ & $17 / 23$ & $14 / 23$ \\
\hline Neuropsychiatric & $7 / 26$ & $4 / 26$ & $11 / 23$ & 9/23 \\
\hline Dysautonomia & $4 / 26$ & $4 / 26$ & $6 / 23$ & $4 / 23$ \\
\hline Gastrointestinal & $8 / 26$ & $5 / 26$ & $5 / 23$ & $5 / 23$ \\
\hline Dermatological & $9 / 26$ & $3 / 26$ & $7 / 23$ & $7 / 23$ \\
\hline ENT & $10 / 26$ & $8 / 26$ & $7 / 23$ & $8 / 23$ \\
\hline $\begin{array}{l}\text { Average symptom } \\
\text { burden per patient } \pm S D\end{array}$ & $4.28 \pm 1.70$ & $2.68 \pm 1.97$ & $4.91 \pm 2.57$ & $4.39 \pm 2.49$ \\
\hline
\end{tabular}

ENT, ear, nose and throat; HRA, histamine receptor antagonist.

87-408 days, asymptomatic controls 100-404 days), we observed marked perturbations in the numbers of circulating EM T cells (automated total lymphocyte counts were normal).

Twenty-five out of 49 symptomatic and 3 out of 16 asymptomatic participants had CD4 + EM counts that were below the lower limit of the normal range (figure $2 \mathrm{~A}$ ), and receiver operating characteristic (ROC) analysis confirmed that the CD4 + EM count could distinguish the two groups (figure 2B). Forty-three out of 49 patients with long COVID and 14 out of 16 of the asymptomatic group had reduced CD8 + EM counts, which were below the median of the normal range (figure 2C). Although the mean count was lower in the long COVID than in the asymptomatic group, the CD8 + EM count did not distinguish the two cohorts in an ROC analysis (figure 2D). All other T cell compartments, including CD4 + and CD8 + central memory (CM) cells, naïve $T$ cells and TEMRA cells, were within normal limits.

Our flow cytometry antibody panel allowed us to compare the expression levels (antigen densities) of proteins important for regulating T cell function. ${ }^{15}$ We observed that the antigen density of PD-1 (programmed cell death protein 1, CD279) was significantly increased in both CD4 + and CD8 + CM cells in all participants, although this was more marked in symptomatic patients with long COVID (figure 3A). Intriguingly, CD28 expression was significantly increased in $\mathrm{CD} 4+\mathrm{CM}$ cells in the asymptomatic group, but not the long COVID group (figure 3B). Expression of both proteins was similar in all other $\mathrm{T}$ cell compartments, and expression of CD57 did not vary significantly.

Although both the numbers of circulating EM T cells and combined PD- 1 and CD28 antigen density in CM cells distinguished asymptomatic from symptomatic participants (figure 2B, online supplemental figure 3), neither predicted responsiveness to HRA (online supplemental figure 4).

\section{DISCUSSION}

Most patients with long COVID have mild or asymptomatic initial infections and are often diagnosed months later. We report clinical and immunologic features in
49 such patients, comparing them with an age-matched cohort who made full recoveries from COVID-19. We documented their symptoms, quantified their $\mathrm{T}$ cell subcompartments and scored their response to HRA .

The majority were younger females with few comorbidities, although $35 \%$ were previously atopic (table 1 ). Many were polysymptomatic, presenting up to 400 days after initial infection. Our key observations are (1) HRA reduces symptom burden in $72 \%$ of patients. (2) CD8 + EM T cells are reduced for up to 400 days following COVID-19 irrespective of symptoms. (3) Long COVID is associated with reduced CD4 + EM T cells. (4) CD4 + and CD8 + CMT cell PD-1 levels are increased following COVID-19 (more marked in long COVID). (5) Increased CD4 + CMCD28 levels correlate with asymptomatic recovery from COVID-19.

The gradual response to HRA argues against a classical anti-anaphylactic mechanism of action. $\mathrm{T}$ cells express both $\mathrm{H} 1$ and $\mathrm{H} 2$ histamine receptors, ${ }^{16}$ and famotidine also desensitizes $\mathrm{T}$ cells through beta-arrestin, ${ }^{17}{ }^{18}$ which suggests that in long COVID, HRA acts through T cells. All symptoms improved except dysautonomia, suggesting that this arises through another mechanism. Indeed, dysautonomia following other viral infections is associated with autoantibodies to adrenergic and cholinergic receptors, ${ }^{19}$ and COVID-19 is linked with autoimmune phenomena. ${ }^{19} 20$

To our knowledge, this is the first report of a rapid turnaround, routine laboratory test detecting persisting abnormalities in the $\mathrm{T}$ cell landscape many months after mild COVID-19. We identify a late, chronic phase of the $\mathrm{T}$ cell response, perhaps linked to that reported in acute infection. ${ }^{21}{ }^{22} \mathrm{CD} 8+\mathrm{EM}$ cells are reduced irrespective of symptoms, which at this stage likely reflects the recovery from COVID-19 itself, rather than the development of long COVID. Consistent with this, dominant CD8 + responses in acute COVID-19 correlate with milder disease, suggesting a protective role through suppression of inflammation. ${ }^{79}$ 


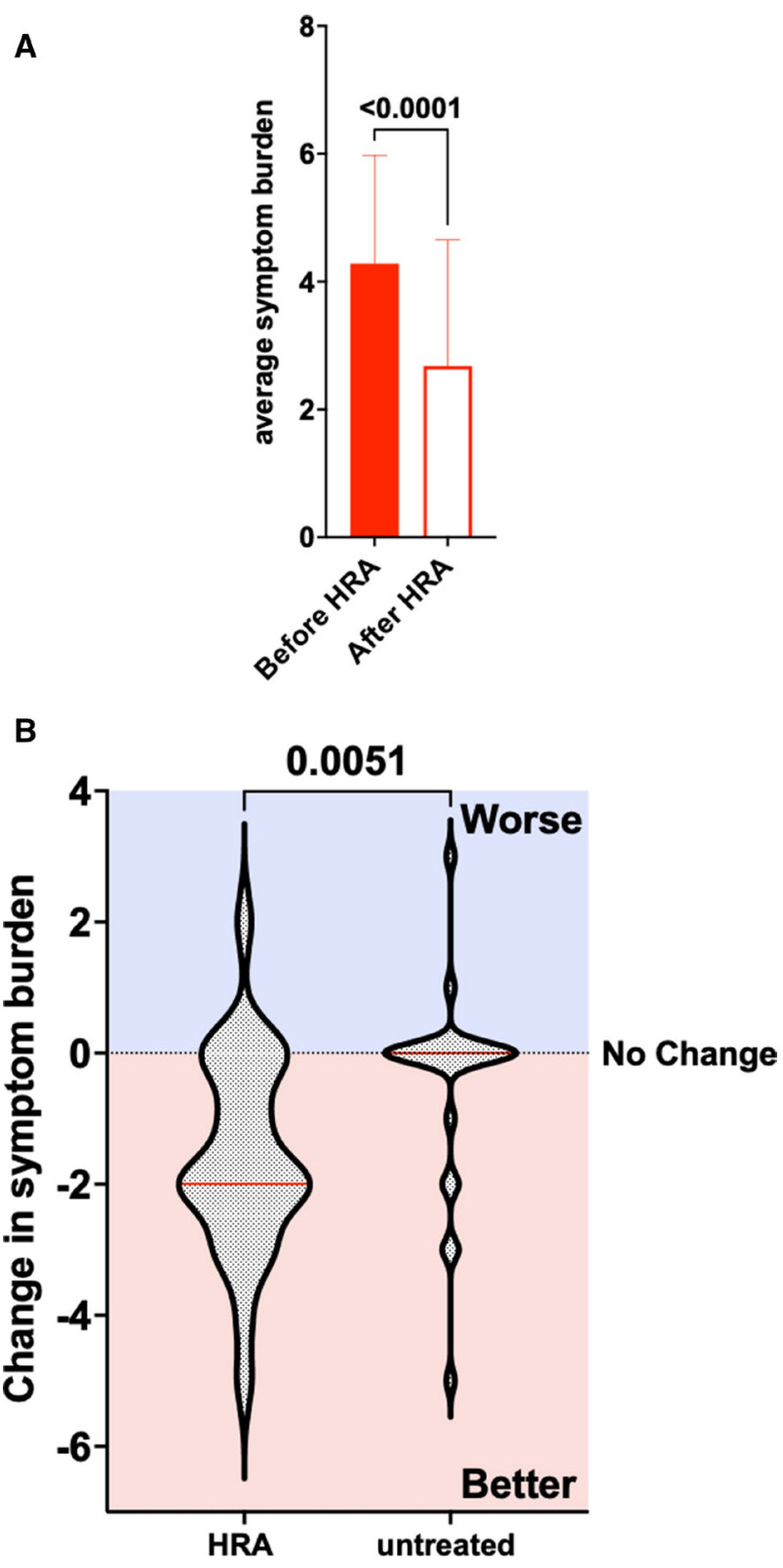

Figure 1 Response to histamine receptor antagonist (HRA) in symptomatic patients. (A) Mean symptom burden $( \pm S D)$ in symptomatic patients before and after HRA treatment $(n=25)$. (B) Change in symptom burden in symptomatic patients who did $(n=25)$ and did not $(n=24)$ receive HRA. Red line denotes median. $p$ values indicated Wilcoxon matched pairs signed-rank test.

T cell changes specific to long COVID include reduced CD4 + EM cells, and increased PD-1 on both CD4 + and CD $8+$ CM cells. PD- 1 is a coinhibitor, contributing to $\mathrm{T}$ cell memory, ${ }^{23}$ and is associated with $\mathrm{T}$ cell exhaustion in chronic viral infection. ${ }^{24}$ Increased CD28, a costimulator essential for signal transduction and $\mathrm{T}$ cell triggering, was limited to those who had made an uneventful recovery from COVID-19 and may represent a 'healthy' immune response to SARS-CoV-2. Our detection of T cell perturbations so late after infection suggests that SARSCoV-2 could persist for longer than originally assumed. In this regard, we note preliminary observations of reverse

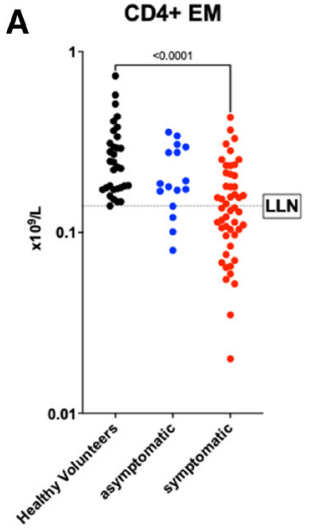

B CD4+ EM symptomatic vs aymptomatic
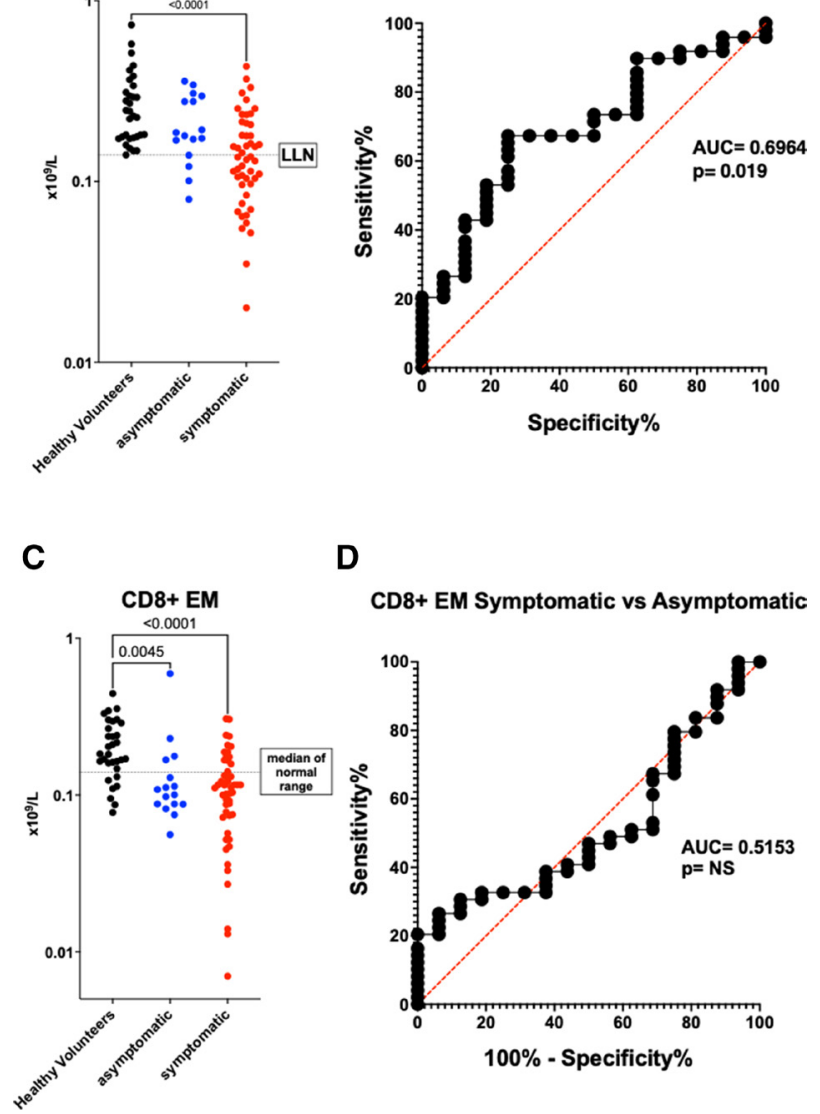

D

CD8+ EM Symptomatic vs Asymptomatic

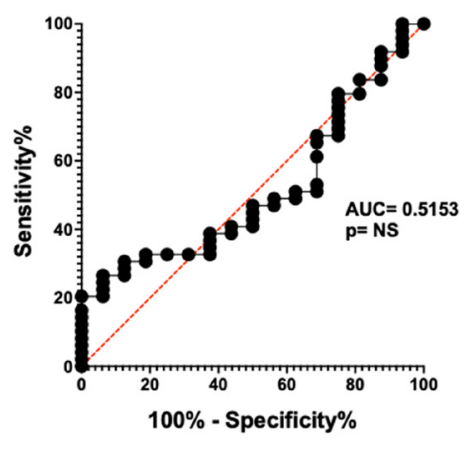

Figure 2 CD4 + and CD8+ effector memory (EM)T cells in patients with long COVID and the asymptomatic fully recovered group. (A) Circulating CD4 + EM T cells $\left(\times 10^{9} / \mathrm{L}\right)$ in healthy volunteers (black), asymptomatic recovered (blue) and symptomatic long COVID (red) participants. Dashed line, LLN (lower limit of normal). Healthy volunteers: mean $0.276 \times 10^{9} / \mathrm{L}$, range $0.140 \times 10^{9} / \mathrm{L}-0.735 \times 10^{9} / \mathrm{L}$. Asymptomatic: mean $0.211 \times 10^{9} / \mathrm{L}$, range $0.079 \times 10^{9} / \mathrm{L}-0.359 \times 10^{9} / \mathrm{L}$. Symptomatic: mean $0.154 \times 10^{9} / \mathrm{L}$, range $0.020 \times 10^{9} / \mathrm{L}-0.433 \times 10^{9} / \mathrm{L}$. p values $<0.05$ indicated Kruskal-Wallis test. (B) Receiver operating characteristic curve of CD4 + EMT cell number in symptomatic (long COVID) and asymptomatic participants. Red dashed line, random classifier. (C) Circulating CD8 + EM T cells $\left(\times 10^{9} / \mathrm{L}\right)$ in healthy volunteers (black), asymptomatic (blue) and symptomatic (red) participants. Dashed line, median of normal range. Healthy volunteers: mean $0.209 \times 10^{9} / \mathrm{L}$, range $0.077 \times 10^{9} / \mathrm{L}-0.444 \times 10^{9} / \mathrm{L}$. Asymptomatic: mean $0.144 \times 10^{9} / \mathrm{L}$, range $0.056 \times 10^{9} / \mathrm{L}-0.594 \times 10^{9} / \mathrm{L}$. Symptomatic: mean $0.117 \times 10^{9} / \mathrm{L}$, range $0.007 \times 10^{9} / \mathrm{L}-0.306 \times 10^{9} / \mathrm{L}$. (D) Receiver operating characteristic curve of CD8 + EMT cell number in symptomatic (long COVID) and asymptomatic participants. Red dashed line, random classifier. AUC, area under curve (c-statistic).

transcription of SARS-CoV-2 RNA and genomic integration in human cells. ${ }^{25}$

Our observations encourage further detailed clinical studies of the potential benefit of HRA and a deeper immunologic analysis of why the $\mathrm{T}$ cell landscape is perturbed in long COVID. Furthermore, $\mathrm{T}$ cell flow cytometry could be developed to provide a rapid and straightforward diagnostic test for long COVID. 

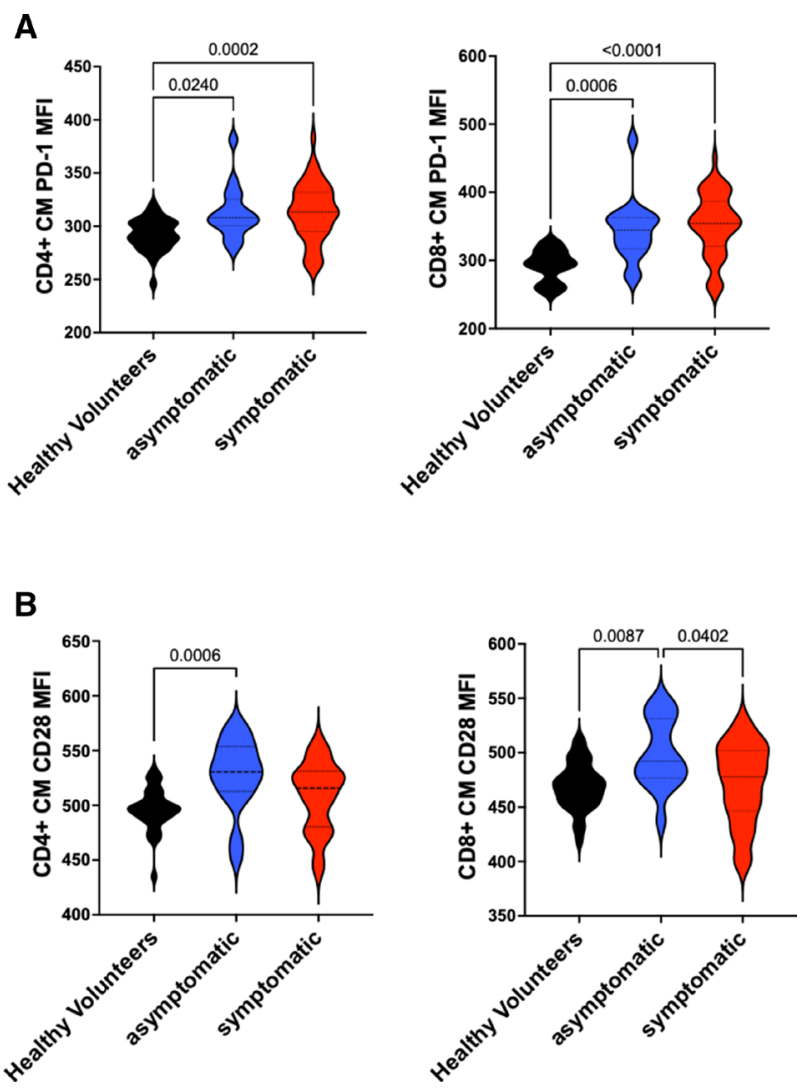

Figure 3 Central memory (CM) T cell programmed cell death protein 1 (PD-1) and CD28 levels distinguish patients with long COVID from the asymptomatic fully recovered group. (A) PD1 antigen density expressed as median fluorescence intensity (MFI, arbitrary units (AU)) in CD4+ (left) and CD8 + CMT cells. CD4 + CM: Healthy volunteers, mean $292 \mathrm{AU}$, range 246-321 AU. Asymptomatic, mean $313 \mathrm{AU}$, range 279-381 AU. Symptomatic, mean $313 \mathrm{AU}$, range 259-383AU. p values $<0.05$ indicated Kruskal-Wallis test. CD8 + CM: Healthy volunteers, mean 292 $\mathrm{AU}$, range 249-329 AU. Asymptomatic, mean $344 \mathrm{AU}$, range 274-477 AU. Symptomatic, mean 352 AU, range 256-451 AU. (B) CD28 antigen density in CD4+ (left) and CD8 + CMT cells. CD4 + CM: Healthy volunteers, mean 496 AU, range 435-532 AU. Asymptomatic: mean 528 AU, range 453-572 AU. Symptomatic: mean 508 AU, 437-563 AU. CD8 + CM: Healthy volunteers: mean $469 \mathrm{AU}$, range 420-512 AU. Asymptomatic: mean $499 \mathrm{AU}$, range 438-547 AU. Symptomatic: mean 472 AU, range 395-532 AU. Significant $p$ values are indicated.

Correction notice This article has been corrected since it was first published to include the Institutional Review Board approval ID.

Contributors PG, VG and RG conceived the project and co-wrote the manuscript. PG saw the patients and documented clinical symptoms. NT collated clinical and laboratory information. RG performed all flow cytometry analysis. RG and VG undertook all statistical analysis.

Funding This study was supported by philanthropic donations from The Dominvs Group (to The Physicians' Clinic) and Sir Peter Wood (to the UCL Cancer Institute), neither of whom contributed either to the design and conduct of the study, or to the analysis of the data. None of the authors received personal funding.

Competing interests None declared.

Patient consent for publication Not applicable.

Ethics approval The study protocol, patient consent form, information leaflets and questionnaire were submitted to the host Institutions' IRB and approved. Patients and volunteers who consented to participate were subsequently fully informed of the analysis results, which were discussed face to face. The Institutional Review Board approval ID is 15:15/12/20.

Provenance and peer review Not commissioned; externally peer reviewed.

Data availability statement All data relevant to the study are included in the article or uploaded as supplementary information.

Supplemental material This content has been supplied by the author(s). It has not been vetted by BMJ Publishing Group Limited (BMJ) and may not have been peer-reviewed. Any opinions or recommendations discussed are solely those of the author(s) and are not endorsed by BMJ. BMJ disclaims all liability and responsibility arising from any reliance placed on the content. Where the content includes any translated material, BMJ does not warrant the accuracy and reliability of the translations (including but not limited to local regulations, clinical guidelines, terminology, drug names and drug dosages), and is not responsible for any error and/or omissions arising from translation and adaptation or otherwise.

Open access This is an open access article distributed in accordance with the Creative Commons Attribution Non Commercial (CC BY-NC 4.0) license, which permits others to distribute, remix, adapt, build upon this work noncommercially, and license their derivative works on different terms, provided the original work is properly cited, an indication of whether changes were made, and the use is non-commercial. See: http://creativecommons.org/ licenses/by-nc/4.0/.

\section{ORCID iD}

Rajeev Gupta http://orcid.org/0000-0001-9573-0505

\section{REFERENCES}

1 Nalbandian A, Sehgal K, Gupta A, et al. Post-Acute COVID-19 syndrome. Nat Med 2021;27:601-15.

2 Augustin M, Schommers P, Stecher M, et al. Post-COVID syndrome in nonhospitalised patients with COVID-19: a longitudinal prospective cohort study. Lancet Reg Health Eur 2021;6:100122.

3 Briggs A, Vassall A. Sci virus dialysis 1, 2020.

4 Gorna R, MacDermott N, Rayner C, et al. Long COVID guidelines need to reflect lived experience. Lancet 2021;397:455-7.

5 Brenu EW, van Driel ML, Staines DR, et al. Immunological abnormalities as potential biomarkers in chronic fatigue Syndrome/Myalgic encephalomyelitis. J Trans/ Med 2011;9:81.

6 Mandarano AH, Maya J, Giloteaux L, et al. Myalgic Encephalomyelitis/chronic fatigue syndrome patients exhibit altered $\mathrm{T}$ cell metabolism and cytokine associations. J Clin Invest 2020;130:1491-505.

7 Sekine T, Perez-Potti A, Rivera-Ballesteros 0 , et al. Robust T cell immunity in convalescent individuals with asymptomatic or mild COVID-19. Cell 2020;183:158-68.

8 Chen Z, John Wherry E. T cell responses in patients with COVID-19. Nat Rev Immunol 2020;20:529-36.

9 Peng Y, Mentzer AJ, Liu G, et al. Broad and strong memory CD4 ${ }^{+}$and CD8 ${ }^{+}$ $\mathrm{T}$ cells induced by SARS-COV-2 in UK convalescent individuals following COVID-19. Nat Immunol 2020;21:1336-45.

10 Dan JM, Mateus J, Kato Y, et al. Immunological memory to SARS-CoV-2 assessed for up to 8 months after infection. Science 2021;371. doi:10.1126/ science.abf4063. [Epub ahead of print: 05 Feb 2021].

11 Daugherty SE, Guo Y, Heath K, et al. Risk of clinical sequelae after the acute phase of SARS-CoV-2 infection: retrospective cohort study. BMJ 2021;373:n1098.

12 Gupta R, Gant VA, Williams B, et al. Increased complement receptor-3 levels in monocytes and granulocytes distinguish COVID-19 patients with pneumonia from those with mild symptoms. Int J Infect Dis 2020;99:381-5.

13 Greenhalgh T, Knight M, A'Court C, et al. Management of post-acute covid-19 in primary care. BMJ 2020;370:m3026.

14 Keswani A, Dhana K, Rosenthal JA, et al. Atopy is predictive of a decreased need for hospitalization for coronavirus disease 2019. Ann Allergy Asthma Immunol 2020;125:479-81.

15 Larbi A, Fulop T. From "truly naïve" to "exhausted senescent" T cells: When markers predict functionality. Cytometry Part A 2014;85:25-35.

16 Sachs B, Hertl M, Merk HF. Histamine receptors on lymphocytes: distribution and functional significance. Skin Pharmacol App/ Skin Physiol 2000;13:313-23.

17 Bond RA, Lucero Garcia-Rojas EY, Hegde A, et al. Therapeutic potential of targeting B-Arrestin. Front Pharmacol 2019;10:124.

18 Alonso N, Zappia CD, Cabrera M, et al. Physiological implications of biased signaling at histamine $\mathrm{H} 2$ receptors. Front Pharmacol 2015;6:45. 
19 Ruzieh M, Batizy L, Dasa O, et al. The role of autoantibodies in the syndromes of orthostatic intolerance: a systematic review. Scand Cardiovasc J 2017:51:243-7.

20 Wallukat G, Hohberger B, Wenzel K, et al. Functional autoantibodies against $\mathrm{G}$-protein coupled receptors in patients with persistent Long-COVID-19 symptoms. J Trans/ Autoimmun 2021;4:100100.

21 Laing AG, Lorenc A, Del Molino Del Barrio I, et al. Author correction: a dynamic COVID-19 immune signature includes associations with poor prognosis. Nat Med 2020;26:1663.

22 Blanco-Melo D, Nilsson-Payant BE, Liu W-C, et al. Imbalanced host response to SARS-CoV-2 drives development of COVID-19. Cell 2020;181:1036-45.
23 Bennett F, Luxenberg $D$, Ling $V$, et al. Program death-1 engagement upon TCR activation has distinct effects on costimulation and cytokine-driven proliferation: attenuation of ICOS, IL-4, and IL-21, but not CD28, IL-7, and IL-15 responses. J Immunol 2003;170:711-8.

24 Jubel JM, Barbati ZR, Burger C, et al. The role of PD-1 in acute and chronic infection. Front Immunol 2020;11:487.

25 Zhang L, Richards A, Barrasa MI, et al. Reverse-transcribed SARS-CoV-2 RNA can integrate into the genome of cultured human cells and can be expressed in patient-derived tissues. Proc Nat/ Acad Sci U S A 2021;118. doi:10.1073/ pnas.2105968118. [Epub ahead of print: 25 May 2021]. 